\title{
PARTIAL DIFFERENTIAL EQUATIONS
}

\section{On One Integral Equation in the Theory of Transform Operators}

\author{
S. M. Sitnik ${ }^{a, *}$ \\ ${ }^{a}$ Belgorod State University, Belgorod, 308002 Russia \\ *e-mail: sitnik@bsu.edu.ru \\ Received February 15, 2020; revised February 15, 2020; accepted April 9, 2020
}

\begin{abstract}
Integral representations of solutions of one differential equation with singularities in the coefficients, containing the Bessel operator perturbed by some potential, are considered. The existence of integral representations of a certain type for such solutions is proved by the method of successive approximations using transform operators. Potentials with strong singularities at the origin are allowed. As compared with the known results, the Riemann function is expressed not via the general hypergeometric function, but, more specifically, via the Legendre function, which helps to avoid unknown constants in the estimates.
\end{abstract}

Keywords: transform operator, Riemann function, Gauss hypergeometric function, Legendre function, singular potential

DOI: $10.1134 / \mathrm{S} 096554252008014 \mathrm{X}$

\section{INTRODUCTION. FORMULATION OF THE PROBLEM}

Consider the problem of constructing an integral representation of a certain kind for solutions of the differential equation

$$
B_{\alpha} u(x)-q(x) u(x)=0, \quad x>0,
$$

where $B_{\alpha}$ is the Bessel operator of the form

$$
B_{\alpha} u=u^{\prime \prime}(x)+\frac{2 \alpha}{x} u^{\prime}(x), \quad \alpha>0, \quad x>0 .
$$

This problem is solved by the method of transform operators. For this, it suffices to construct a Poissontype transform operator $\mathbf{P}_{\alpha}$ of the form

$$
\mathbf{P}_{\alpha} u(x)=u(x)+\int_{x}^{\infty} P(x, t) u(t) d t
$$

with some kernel $P(x, t)$ that interwines the operators $B_{\alpha}$ and $B_{\alpha}-q(x)$ according to the formula

$$
B_{\alpha} \mathbf{P}_{\alpha} u=\mathbf{P}_{\alpha}\left(B_{\alpha}-q(x)\right) u
$$

on functions $u \in C^{2}(0, \infty)$. As a result, we obtain a formula expressing the solutions of Eq. (1) with a spectral parameter, which has the form

$$
B_{\alpha} u(x)-q(x) u(x)=\lambda^{2} u(x), \quad x>0,
$$

via the solutions of the unperturbed equation, i.e., via Bessel functions. In this case, the spectral parameter $\lambda$ does not affect the form of linear transform operators whose kernels do not depend on it. This approach reflects one of the main applications of transform operators: the expression of solutions of more complex differential equations via similar simpler ones.

The theory of transform operators is an important branch of modern mathematics, which has numerous applications (see [1-6]). The possibility of representation (3) with a sufficiently "good" kernel $P$ for a wide class of potentials $q(x)$ underlies the classical methods for solving inverse problems in the quantum scattering theory [7, 8]. For Sturm-Liouville equations, transform operators of form (3) were first constructed by B.Ya. Levin (see [1-6]). 
The transform operators for the Bessel operator of the Sonin and Poisson's type were introduced by Delsart; for the first time, the theory of them was presented in Russian and developed in the famous work by Levitan [9]. Later, in a number of works, transform operators with property (4) for variable potentials were also considered, simultaneously with Sonin-type transform operators $S_{\alpha}$ inverse of (3), satisfying the intertwining relation

$$
S_{\alpha}\left(B_{\alpha}-q(x)\right) u=B_{\alpha} S_{\alpha} u
$$

on appropriate functions.

An original technique for constructing transform operators for a perturbed Bessel equation on the halfaxis was developed by Stashevskaya [10], which allowed her to include into consideration singular potentials with an estimate at zero $|q(x)| \leq c x^{-3 / 2+\varepsilon}, \varepsilon>0$; for integer $\alpha$, this technique was subsequently widely developed. The case of continuous $q, \alpha>0$, was thoroughly considered by Sokhin [11,12], as well as other authors (for details, see [5, 6]). Transform operators with "bad" potentials: distributions from a certain class, were considered in [8]. V.V. Kravchenko developed a special method for representing the kernels of transform operators in the form of series: Spectral Parameter Power Series (SPPS) method [13, 14]; this method proved to be well suited for numerical solution of applied problems and computer modeling, including solving direct and inverse spectral problems $[15,16]$. The author's results for some special cases of the considered problem of constructing transform operators (3) and (4) were previously published in [17, 18].

In many mathematical and physical problems, it is necessary to consider strongly singular potentials, e.g., admitting an arbitrary power singularity at zero. In this paper, the results on the integral representation of solutions of equations with such singular potentials are formulated. From the potential it is only required that the potential be majorized by a certain function summable at infinity. In particular, in this paper, the class of admissible potentials includes the singular potential $q=x^{-2}$, the strongly singular potential with a power singularity $q=x^{-2-\varepsilon}, \varepsilon>0$, Yukawa-type potentials $q=e^{-\alpha x} / x$, Bargman and Batman-Chadan potentials [7], and others. No additional conditions are imposed on the function $q(x)$, such as fast oscillations at the origin or sign constancy, which makes it possible to study attractive and repulsive potentials using the same method. The absence of limiting conditions at zero is the advantage of consideration of Levin-type transform operators (3).

The main objective of this article is to study the integral equation for the kernel of transform operator (3). After reducing the problem to an integral equation, the existence and uniqueness of the solution, as well as its necessary smoothness, are proved. Estimates of the solution are obtained in terms of the parameter and the potential of the original equation (1), which are expressed via Legendre functions. Simpler estimates are obtained for a special class of power-law potentials. This work uses a technique based on the application of the Riemann function to the Euler-Poisson-Darboux equation, estimates of integrals using the Mellin transform, and the Slater-Marichev theorem.

It should be noted that, in this work, transform operators of a special type, differing from previously known in some details, are constructed. For this, only the cases of equal limits (of types $[0 ; a]$ or $[a ; \infty]$ ) in the main integral equation for the kernel of the transform operator were considered. In this paper, it is shown that the cases of different limits in the main integral equation can also be considered. It is precisely this arrangement of limits that made it possible to cover a wider class of potentials with singularities at zero. In addition, in comparison with reasoning by analogy with the classical work of Levitan [9], we supplement and improve this scheme by considering a modified integral equation for the kernel of the transform operator. As it turned out, the Riemann function used in the proof can be expressed not only via the general Gauss hypergeometric function with three parameters, but also, more specifically, via the Legendre function with two parameters, which allows one to get rid of the indefinite constants in the estimates from previous works.

\section{SOLUTION OF THE MAIN INTEGRAL EQUATION FOR THE KERNEL OF A TRANSFORM OPERATOR}

Let us introduce new variables and functions using the formulas:

$$
\begin{gathered}
\xi=\frac{t+x}{2}, \quad \eta=\frac{t-x}{2}, \quad \xi \geq \eta>0 \\
K(x, t)=\left(\frac{x}{t}\right)^{\alpha} P(x, t), \quad u(\xi, \eta)=K(\xi-\eta, \xi+\eta) .
\end{gathered}
$$


Let $v=\alpha-1$. Thus, to justify representation (3) of the solution of Eq. (1), it suffices to determine the function $u(\xi, \eta)$. It is known $[17,18]$ that, if there exists a twice continuously differentiable solution $u(\xi, \eta)$ of the integral equation

$$
u(\xi, \eta)=-\frac{1}{2} \int_{\xi}^{\infty} R_{v}(s, 0 ; \xi, \eta) q(s) d s-\int_{\xi}^{\infty} d s \int_{0}^{\eta} q(s+\tau) R_{v}(s, \tau ; \xi, \eta) u(s, \tau) d \tau,
$$

under the conditions $0<\tau<\eta<\xi<s$, then the sought-for function $P(x, t)$ is determined by formulas (5) via this solution $u(\xi, \eta)$. The function $R_{v}=R_{\alpha-1}$ is the Riemann function, which occurs when solving a certain Goursat problem for a singular inhomogeneous hyperbolic equation of the form (one of the forms of the Euler-Poisson-Darboux equation)

$$
\frac{\partial^{2} u(\xi, \eta)}{\partial \xi \partial \eta}+\frac{4 \alpha(\alpha-1) \xi \eta}{\left(\xi^{2}-\eta^{2}\right)^{2}} u(\xi, \eta)=f(\xi, \eta)
$$

which, in our case, can be transformed to

$$
\frac{\partial^{2} u(\xi, \eta)}{\partial \xi \partial \eta}+\frac{4 \alpha(\alpha-1) \xi \eta}{\left(\xi^{2}-\eta^{2}\right)^{2}} u(\xi, \eta)=q(\xi+\eta) u(\xi, \eta)
$$

This Riemann function is known explicitly (see $[9,19])$; it is expressed in terms of the Gauss hypergeometric function ${ }_{2} F_{1}$ by the formula

$$
R_{v}=\left(\frac{s^{2}-\eta^{2}}{s^{2}-\tau^{2}} \frac{\xi^{2}-\tau^{2}}{\xi^{2}-\eta^{2}}\right)_{2}^{v} F_{1}\left(-v,-v ; 1 ; \frac{s^{2}-\xi^{2}}{s^{2}-\eta^{2}} \frac{\eta^{2}-\tau^{2}}{\xi^{2}-\tau^{2}}\right) .
$$

This expression is simplified in [17], and it is shown that the Riemann function in this case is expressed in terms of the Legendre function by the formula

$$
R_{v}(s, \tau, \xi, \eta)=P_{v}\left(\frac{1+A}{1-A}\right), \quad A=\frac{\eta^{2}-\tau^{2}}{\xi^{2}-\tau^{2}} \frac{s^{2}-\xi^{2}}{s^{2}-\eta^{2}}
$$

The main result of this article is the following theorem.

Theorem 1. Let a function $q(r) \in C^{1}(0, \infty)$ satisfy the condition

$$
|q(s+\tau)| \leq|p(s)| \quad \forall s, \quad \forall \tau, \quad 0<\tau<s, \quad \int_{\xi}^{\infty}|p(t)| d t<\infty \quad \forall \xi>0 .
$$

Then, there exists an integral representation of the form (3), whose kernel satisfies the estimate

$$
\begin{aligned}
& |P(r, t)| \leq\left(\frac{t}{r}\right)^{\alpha} \frac{1}{2} \int_{\frac{t+r}{2}}^{\infty} P_{\alpha-1}\left(\frac{y^{2}\left(t^{2}+r^{2}\right)-\left(t^{2}-r^{2}\right)}{2 t r y^{2}}\right)|p(y)| d y \\
& \times \exp \left[\left(\frac{t-r}{2}\right) \frac{1}{2} \int_{\frac{t+r}{2}}^{\infty} P_{\alpha-1}\left(\frac{y^{2}\left(t^{2}+r^{2}\right)-\left(t^{2}-r^{2}\right)}{2 t r y^{2}}\right)|p(y)| d y\right] .
\end{aligned}
$$

In this case, the kernel $P(x, t)$ of the transform operator, as well as the solution of Eq. (1), are twice continuously differentiable with respect to their arguments separately on the corresponding domains of definition.

Proof. The proof of the main Theorem 1 is divided into a series of lemmas.

Let us introduce the notation

$$
\begin{gathered}
I_{q}(\xi, \eta)=\frac{1}{2} \int_{\xi}^{\infty} R_{v}(y, 0 ; \xi, \eta)|p(y)| d y=\frac{1}{2} \int_{\xi}^{\infty} P_{v}\left(\frac{y^{2}\left(\xi^{2}+\eta^{2}\right)-2 \xi^{2} \eta^{2}}{y^{2}\left(\xi^{2}-\eta^{2}\right)}\right)|p(y)| d y, \\
u_{0}(\xi, \eta)=-\frac{1}{2} \int_{\xi}^{\infty} R_{v}(s, 0 ; \xi, \eta)|p(s)| d s,
\end{gathered}
$$




$$
\mathbf{A} u_{0}(\xi, \eta)=-\int_{\xi}^{\infty} d s \int_{0}^{\eta} q(s+\tau) R_{v}(s, \tau ; \xi, \eta) u_{0}(s, \tau) d \tau .
$$

We will prove the uniform convergence of the operator Neumann series

$$
\sum_{k=0}^{\infty} \mathbf{A}^{k} u_{0}(\xi, y)
$$

and the possibility of its double differentiation.

Lemma 1. The following estimate is true:

$$
\left|u_{0}(\xi, \eta)\right| \leq I_{q}(\xi, \eta) .
$$

The proof immediately follows from definition (9).

Lemma 2. Let $0<\tau<\eta<\xi<s$. Then, we have the inequality

$$
I_{q}(s, t) \leq I_{q}(\xi, \eta) \text {. }
$$

Proof. By the assumption, $0<\tau<\eta<\xi<s<y$. Let us then show that

$$
\frac{\tau^{2}}{s^{2}} \frac{\left(y^{2}-s^{2}\right)}{\left(y^{2}-\tau^{2}\right)} \leq \frac{\eta^{2}}{\xi^{2}} \frac{\left(y^{2}-\xi^{2}\right)}{\left(y^{2}-\eta^{2}\right)}
$$

Indeed, this inequality is equivalent to

$$
\tau^{2} \xi^{2}\left(y^{2}-s^{2}\right)\left(y^{2}-\eta^{2}\right) \leq \eta^{2} s^{2}\left(y^{2}-\xi^{2}\right)\left(y^{2}-\tau^{2}\right),
$$

which is obvious, since each of the factors on the left does not exceed the corresponding factor on the right. Next, consider the function at $0<x<1$ :

$$
f(x)=\frac{1+x}{1-x} \geq 1, \quad f^{\prime}(x)=\frac{2}{(1-x)^{2}}>0, \quad 0<x<1 .
$$

Therefore, this function increases in $x$. Therefore,

$$
\frac{1+\frac{\tau^{2}}{s^{2}} \frac{\left(y^{2}-s^{2}\right)}{\left(y^{2}-\tau^{2}\right)}}{1-\frac{\tau^{2}}{s^{2}} \frac{\left(y^{2}-s^{2}\right)}{\left(y^{2}-\tau^{2}\right)}} \leq \frac{1+\frac{\eta^{2}}{\xi^{2}} \frac{\left(y^{2}-\xi^{2}\right)}{\left(y^{2}-\eta^{2}\right)}}{1-\frac{\eta^{2}}{\xi^{2}} \frac{\left(y^{2}-\xi^{2}\right)}{\left(y^{2}-\eta^{2}\right)}} .
$$

The Legendre function $P_{v}(x)$ with $v>-1$ monotonically increases on the interval $x \in(1, \infty)$ and $P_{v}(x)>1$. Therefore,

$$
P_{v}\left(\frac{1+\frac{\tau^{2}}{s^{2}} \frac{\left(y^{2}-s^{2}\right)}{\left(y^{2}-\tau^{2}\right)}}{1-\frac{\tau^{2}}{s^{2}} \frac{\left(y^{2}-s^{2}\right)}{\left(y^{2}-\tau^{2}\right)}}\right) \leq P_{v}\left(\frac{1+\frac{\eta^{2}}{\xi^{2}} \frac{\left(y^{2}-\xi^{2}\right)}{\left(y^{2}-\eta^{2}\right)}}{1-\frac{\eta^{2}}{\xi^{2}} \frac{\left(y^{2}-\xi^{2}\right)}{\left(y^{2}-\eta^{2}\right)}}\right) .
$$

This inequality can be written as

$$
P_{v}\left(\frac{y^{2}\left(s^{2}+\tau^{2}\right)-2 s^{2} \tau^{2}}{y^{2}\left(s^{2}-\tau^{2}\right)}\right) \leq P_{v}\left(\frac{y^{2}\left(\xi^{2}+\eta^{2}\right)-2 \xi^{2} \eta^{2}}{y^{2}\left(\xi^{2}-\eta^{2}\right)}\right) .
$$

Note that we have actually proved the inequality for the Riemann function,

$$
R_{v}(y, 0 ; s, \tau) \leq R_{v}(y, 0 ; \xi, \eta),
$$

under conditions $0<\tau<\eta<\xi<s<y$.

From the above calculations, we obtain the estimate

$$
I_{q}(s, \tau)=\frac{1}{2} \int_{s}^{\infty} R_{v}(y, 0 ; s, \tau)|p(y)| d y \leq \frac{1}{2} \int_{\xi}^{\infty} R_{v}(y, 0 ; s, \tau)|p(y)| d y .
$$


Replacing the lower limit of integration $s$ with $\xi<s$, we can only increase the value of the integral, since the Riemann function is positive, $R_{v}>0$. As a result, we arrive at estimate (11).

The lemma is proved.

Lemma 3. The nth term of Neumann series (10) is estimated as

$$
\left|u_{n}(\xi, \eta)\right| \leq I_{q}(\xi, \eta) \frac{\left[\eta I_{q}(\xi, \eta)\right]^{n}}{n !} .
$$

Proof. The lemma is proved by induction. For $n=0$, inequality (13) reduces to the already proved inequality from Lemma 1. Let (13) hold for some $n=k$. Then, for the next term of the Neumann series, we obtain

$$
\begin{aligned}
& \left|u_{k+1}(\xi, \eta)\right| \leq\left|\int_{\xi}^{\infty} d s \int_{0}^{\eta} R_{v}(s, \tau ; \xi, \eta) u_{k}(s, \tau) q(s+\tau) d \tau\right| \\
\leq & \int_{\xi}^{\infty} d s \int_{0}^{\eta} R_{v}(s, \tau ; \xi, \eta)|q(s+\tau)| I_{q}(s, \tau) \frac{\left[\eta I_{q}(s, \tau)\right]^{k}}{k !} d \tau .
\end{aligned}
$$

Repeating the reasoning from the proof of the previous lemma, we obtain

$$
R_{v}(s, \tau ; \xi, \eta) \leq R_{v}(s, 0 ; \xi, \eta)
$$

since

$$
R_{v}(s, \tau ; \xi, \eta)=P_{v}\left(\frac{1+A}{1-A}\right), \quad A=\frac{\eta^{2}-\tau^{2}}{\xi^{2}-\tau^{2}} \frac{s^{2}-\xi^{2}}{s^{2}-\eta^{2}},
$$

and the maximum of $A$ with respect to $\tau$ is achieved at $\tau=0$. Given inequality (14) and the assumed inequality (13), we arrive at the estimate

$$
\left|u_{k+1}(\xi, \eta)\right| \leq I_{q}(\xi, \eta) \frac{\left[\tau I_{q}(\xi, \eta)\right]^{k}}{k !} \int_{\xi}^{\infty} R_{v}(s, 0 ; \xi, \eta) \int_{0}^{\eta}|q(s+\tau)| \tau^{k} d \tau d s .
$$

We consider potentials satisfying the inequality $|q(s+\tau)| \leq|p(s)|, 0<\tau<s$. We finally have

$$
\left|u_{k+1}(\xi, \eta)\right| \leq I_{q}(\xi, \eta) \frac{\left[I_{q}(\xi, \eta)\right]^{k+1}}{k !} \frac{\eta^{k+1}}{(k+1)},
$$

which proves estimate (13) for all $n$.

The lemma is proved.

Now let us complete the proof of Theorem 1. Summing up all estimates (13), we find that the Neumann series converges uniformly in the domain $0<\eta<\xi$ and its sum is a continuous function satisfying the inequality

$$
|u(\xi, \eta)| \leq I_{q}(\xi, \eta) \exp \left[\eta I_{q}(\xi, \eta)\right] .
$$

It follows from (15) that we could prove the convergence of series (10) for a summable potential $q$ that can be approximated by continuous potentials.

Returning to the functions $K$ and $P$, we obtain the inequalities

$$
\begin{gathered}
|K(x, t)| \leq I_{q}\left(\frac{t+x}{2}, \frac{t-x}{2}\right) \exp \left[\left(\frac{t-x}{2}\right) I_{q}\left(\frac{t+x}{2}, \frac{t-x}{2}\right)\right], \\
|P(x, t)| \leq\left(\frac{t}{x}\right)^{\alpha} I_{q}\left(\frac{t+x}{2}, \frac{t-x}{2}\right) \exp \left[\left(\frac{t-x}{2}\right) I_{q}\left(\frac{t+x}{2}, \frac{t-x}{2}\right)\right] .
\end{gathered}
$$

The quantity $I_{q}$ entering into the estimates is transformed as

$$
I_{q}\left(\frac{t+x}{2}, \frac{t-x}{2}\right)=\frac{1}{2} \int_{\frac{t+x}{2}}^{\infty} P_{\alpha-1}\left(\frac{y^{2}\left(t^{2}+x^{2}\right)-\left(t^{2}-x^{2}\right)}{2 t x y^{2}}\right)|p(y)| d y .
$$


Thus, we arrive at estimate (9).

To complete the proof of Theorem 1, it only remains to justify the existence of second continuous derivatives of the function $P(x, t)$ with respect to the variables $x, t$ under the condition $q \in C^{1}(x>0)$. Obviously, it is equivalent to the existence of second continuous derivatives of the function $u(\xi, \eta)$ with respect to the variables $\xi, \eta$. The last statement is proved using the technique presented above, by the method of successive approximations, and completely repeats the corresponding fragment of the proof from [17].

The theorem is proved.

Let us list the classes of potentials for which conditions (8) are satisfied. If $|q(x)|$ decreases monotonically, we can accept $p(x)=|q(x)|$. For potentials with an arbitrary singularity at the origin and increasing at $0<x<M$ (e.g., the Coulomb potentials $q=-\frac{1}{x}$ ), which are truncated by zero at infinity, $q(x)=0$, $x>M$, we can accept $p(x)=|q(M)|, x<M, p(x)=0, x \geq M$. Condition (8) will also be satisfied by potentials with an estimate $q(x+\tau) \leq c|q(x)|=|p(x)|$. The possibility of such strengthening of Theorem 1 was noted by V.V. Katrakhov.

In particular, the above conditions are satisfied by the following potentials, occurring in applications: a strongly singular potential with a power singularity of the form $q(x)=x^{-2-\varepsilon}$, various Bargman potentials:

$$
q_{1}(x)=-\frac{e^{-a x}}{\left(1+\beta e^{-a x}\right)^{2}}, \quad q_{2}(x)=\frac{c_{2}}{\left(1+c_{3} x\right)^{2}}, \quad q_{3}(x)=\frac{c_{4}}{c h^{2}\left(c_{5} x\right)},
$$

and Yukawa potentials:

$$
q_{4}(x)=-\frac{e^{-a x}}{x}, \quad q_{5}(x)=\int_{x}^{\infty} e^{-a t} d c(t)
$$

(see, e.g., [7]).

Remark. In fact, the proof of the above theorem does not employ the explicit form of the Riemann function (7). We only use the existence of the Riemann function, its positivity, and some special property of monotonicity (14). These facts are rather general; therefore, the results can be extended to a fairly wide class of differential equations.

The estimate from Theorem 1 for potentials of a general form can be transformed into a less accurate, but more conceivable estimate.

Theorem 2. Let the conditions of Theorem 1 be satisfied. Then, the kernel $P(x, t)$ of a transform operator satisfies the estimate

$$
|P(x, t)| \leq \frac{1}{2}\left(\frac{t}{x}\right)^{\alpha} P_{\alpha-1}\left(\frac{t^{2}+x^{2}}{2 t x}\right) \int_{x}^{\infty}|p(y)| d y \exp \left[\frac{1}{2}\left(\frac{t-x}{2}\right) P_{\alpha-1}\left(\frac{t^{2}+x^{2}}{2 t x}\right) \int_{x}^{\infty}|p(y)| d y\right] .
$$

Note that, as $x \rightarrow 0$, the kernel of the integral representation can have an exponential singularity.

\section{ESTIMATES FOR THE CASE OF A POTENTIAL WITH A POWER SINGULARITY AT ZERO}

For the class of potentials with a power singularity of the form

$$
q(x)=x^{-(2 \beta+1)}, \quad \beta>0,
$$

estimates obtained above can be simplified without reducing their accuracy. The restriction on $\beta$ is caused by the condition of summability at infinity.

Theorem 3. Consider a potential of the form (16). Then, Theorem 1 holds with the estimate

$$
|P(x, t)| \leq\left(\frac{t}{x}\right)^{\alpha} \frac{\Gamma(\beta) 4^{\beta-1}}{\left(t^{2}-x^{2}\right)^{\beta}} P_{\alpha-1}^{-\beta}\left(\frac{t^{2}+x^{2}}{2 t x}\right) \exp \left[\left(\frac{t-x}{x}\right) \frac{\Gamma(\beta) 4^{\beta-1}}{\left(t^{2}-x^{2}\right)^{\beta}} P_{\alpha-1}^{-\beta}\left(\frac{t^{2}+x^{2}}{2 t x}\right)\right],
$$

where $P_{v}^{\mu}(\cdot)$ is the Legendre function [20], the quantity $\beta$ is determined from (16), and $\alpha$ is determined from (2) 
Before starting the proof, it should be noted that this estimate is obtained after rather tedious calculations using the famous Slater-Marichev theorem [21], which helps to calculate the necessary integrals in terms of hypergeometric functions after reducing them the Mellin convolution.

Proof. For this class of potentials, we will simplify estimate (15), which constitutes the content of Theorem 1 , without reducing its accuracy. To that end, we will calculate explicitly the quantity $I_{q}$ entering into estimate (15). The proof of Theorem 3 is divided into two lemmas.

Lemma 4. For a potential of the form (16), we have the relationship

$$
I_{q}(\xi, \eta)=\frac{1}{4 \xi^{2 \beta}} \int_{0}^{1} P_{v}(2 \alpha z+1)(1-z)^{\beta-1} d z,
$$

where $P_{v}$ is the Legendre function and $\alpha=\eta^{2} /\left(\xi^{2}-\eta^{2}\right)$.

Proof. Consider the quantity

$$
I_{q}(\xi, \eta)=\frac{1}{2} \int_{\xi}^{\infty} P_{v}\left(\frac{t^{2}\left(\xi^{2}+\eta^{2}\right)-2 \xi^{2} \eta^{2}}{t^{2}\left(\xi^{2}-\eta^{2}\right)}\right) \frac{d t}{t^{2 \beta+1}}
$$

Perform the change of variables, denoting the argument of the Legendre function by $x$ :

$$
x=\frac{t^{2}\left(\xi^{2}+\eta^{2}\right)-2 \xi^{2} \eta^{2}}{t^{2}\left(\xi^{2}-\eta^{2}\right)}, \quad d x=\frac{4 \xi^{2} \eta^{2}}{t^{3}\left(\xi^{2}-\eta^{2}\right)} d t .
$$

With this replacement, the new limits of integration are the numbers

$$
1, \quad 1+\frac{2 \eta^{2}}{\xi^{2}-\eta^{2}}=\frac{\xi^{2}+\eta^{2}}{\xi^{2}-\eta^{2}}=B>1,
$$

and the variable $t$ is calculated by the formula

$$
t=\xi \eta\left(\frac{2}{\xi^{2}+\eta^{2}-x\left(\xi^{2}-\eta^{2}\right)}\right)^{1 / 2} .
$$

This leads us to the following expression for $I_{q}$ :

$$
I_{q}(\xi, \eta)=\frac{1}{2} \int_{1}^{B} P_{v}(x) \frac{t^{3}\left(\xi^{2}-\eta^{2}\right)}{4 \xi^{2} \eta^{2}} \frac{d t}{t^{2 \beta+1}}=\frac{1}{2} \int_{1}^{B} P_{v}(x)\left[\frac{\xi^{2}-\eta^{2}}{4 \xi^{2} \eta^{2}}\right]\left[\frac{\xi^{2}+\eta^{2}-x\left(\xi^{2}-\eta^{2}\right)}{2 \xi^{2} \eta^{2}}\right]^{\beta-1} d x .
$$

In the last integral, we perform another change of variable by the formula

$$
z=(x-1) \frac{\xi^{2}-\eta^{2}}{2 \eta^{2}}, \quad\left(d z=\frac{\xi^{2}-\eta^{2}}{2 \eta^{2}}\right) d x .
$$

As a result, we have

$$
\begin{gathered}
I_{q}(\xi, \eta)=\frac{1}{2}\left(\frac{\xi^{2}-\eta^{2}}{4 \xi^{2} \eta^{2}}\right) \int_{0}^{1} P_{v}(2 \alpha z+1) \frac{2 \eta^{2}}{\xi^{2}-\eta^{2}} \\
\times\left[\frac{\xi^{2}+\eta^{2}-\left(\xi^{2}-\eta^{2}\right)\left(\frac{2 \eta^{2}}{\xi^{2}-\eta^{2}} z+1\right)}{2 \xi^{2} \eta^{2}}\right] d z=\frac{1}{4 \xi^{2 \beta}} \int_{0}^{1} P_{v}(2 \alpha z+1)(1-z)^{\beta-1} d z,
\end{gathered}
$$

where $\alpha=\eta^{2} /\left(\xi^{2}-\eta^{2}\right)$. We have obtained formula (17).

The lemma is proved.

Lemma 5. Let the conditions $a>0$ and $\beta>0$ be satisfied. Then, we have the formula:

$$
\int_{0}^{1} P_{v}(2 \alpha x+1)(1-x)^{\beta-1} d x=\Gamma(\beta)\left[\frac{1+\alpha}{\alpha}\right]^{\beta / 2} P_{v}^{-\beta}(2 \alpha+1) .
$$


Proof. The proof uses the notation and a technique based on the Slater-Marichev theorem [21].

Making the change of variables $t=1 / x$ in the integral in (18), we obtain

$$
\int_{0}^{1} P_{v}(2 \alpha x+1)(1-x)^{\beta-1} d x=\int_{1}^{\infty} P_{v}\left(2 \frac{\alpha}{t}+1\right)(t-1)^{\beta-1} t^{-\beta} \frac{d t}{t}=\int_{0}^{\infty} P_{v}\left(2 \frac{\alpha}{t}+1\right)(t-1)_{+}^{\beta-1} t^{-\beta} \frac{d t}{t}=I(\alpha),
$$

where the truncated power function is denoted as $x_{+}^{\lambda}$. We apply to the function $I(\alpha)$ the Mellin transform with respect to the variable $\alpha(\alpha>0)$. Using the Mellin convolution theorem [21], we obtain:

$$
M[I(\alpha)](s)=M\left[P_{v}(2 x+1)\right](s) M\left[x^{-\beta}(x-1)_{+}^{\beta-1}\right](s) .
$$

Using successively relationships 6 (1), (4), and 2 (4) from [21], we arrive at the expression

$$
\begin{gathered}
M[I(\alpha)](s)=-\frac{\sin \pi v}{\pi} \frac{\Gamma(s) \Gamma(-v-s) \Gamma(1+v-s) \Gamma(\beta) \Gamma(1-s)}{\Gamma(1-s) \Gamma(1+\beta-s)} \\
=-\frac{\sin \pi v}{\pi} \Gamma(\beta) \Gamma\left[\begin{array}{cc}
s, & -v-s, \\
1+\beta-s
\end{array}\right],
\end{gathered}
$$

where the Slater designation is used for the ratio of the products of gamma functions. In the notation of the Slater-Marichev theorem, we have

$$
\begin{gathered}
(a)=(0), \quad(b)=(-v, 1+v), \quad(c)=\varnothing, \quad(d)=(1+\beta), \\
A=1, \quad B=2, \quad C=0, \quad D=1 .
\end{gathered}
$$

Applying the Slater-Marichev theorem, we obtain formulas for $I(\alpha)$ at $0<\alpha<1$ :

$$
I(\alpha)=-\frac{\sin \pi v}{\pi} \frac{\Gamma(1+v) \Gamma(-v)}{\Gamma(1+\beta)}{ }_{2} F_{1}(-v, 1+v ; 1+\beta ;-\alpha)=\Gamma(\beta) \alpha^{-\beta / 2}(1+\alpha)^{\beta / 2} P_{v}^{-\beta}(1+2 \alpha),
$$

where we used formula (3) from [20; p. 126], and an identity for gamma functions (see [20]):

$$
\Gamma(-v)=\frac{\pi}{v \Gamma(v) \sin \pi v}
$$

For $\alpha \geq 1$, we obtain an expression of different form:

$$
\begin{aligned}
I(\alpha)= & -\frac{\sin \pi v}{\pi} \Gamma(\beta)\left\{\alpha^{v} \Gamma\left[\begin{array}{c}
1+v+v, \\
1+\beta+v
\end{array}\right]{ }_{2} F_{1}\left(-v, 1-1-\beta-v ; 1-1-v-v ;-\frac{1}{\alpha}\right)\right. \\
& \left.+\alpha^{-1-v} \Gamma\left[\begin{array}{c}
-v-1-v, 1+v \\
1+\beta-1-v
\end{array}\right]{ }_{2} F_{1}\left(1+v, 1-1-\beta+1+v ; 1+v ;-\frac{1}{\alpha}\right)\right\} \\
& =-\frac{\sin \pi v}{\pi} \Gamma(\beta)\left\{\alpha^{v} \frac{\Gamma(2 v+1) \Gamma(-v)}{\Gamma(1+\beta+v)}{ }_{2} F_{1}\left(-v,-\beta-v ;-2 v ;-\frac{1}{\alpha}\right)\right. \\
& \left.+\alpha^{-1-v} \frac{\Gamma(-1-2 v) \Gamma(1+v)}{\Gamma(\beta-v)}{ }_{2} F_{1}\left(1+v, 1+v-\beta ; 1+v ;-\frac{1}{\alpha}\right)\right\} .
\end{aligned}
$$

But, according to [20, p. 131, formula (19)], the expressions obtained for $I(\alpha)$ with $0<\alpha<1$ and $\alpha \geq 1$ coincide.

We see from (19) that we have obtained the desired formula (18); however, its derivation is not completely rigorous, since we did not check the admissibility of applying the Mellin transform and the conditions of the Slater-Marichev theorem (in our case, rather complicated). However, now we can apply the Mellin transform to both sides of the formal equality (18) obtained. As a result, we prove that, for $\alpha>0$ and $\beta>0$, relationship (18) is an identity.

The lemma is proved.

As an implication, we now obtain the necessary estimate for Theorem 3.

The theorem is proved. 
The simplest similar estimate was obtained in [17] for the potential $q(x)=c x^{-2}$, for which $\beta=\frac{1}{2}$. As follows from [20], in this case, the Legendre function $P_{v}^{-1 / 2}(z)$ can be expressed via elementary functions. Therefore, the corresponding estimate can be expressed in terms of elementary functions, too.

Another potential for which the estimate obtained can be simplified even more and expressed in terms of elementary functions is a potential of the form $q(x)=x^{-(2 \beta+1)}$ when the parameters are related as $\beta=\alpha-1$.

Corollary. Let the parameters be related as $\beta=\alpha-1$. Then, the estimate from Theorem 3 takes the form

$$
\begin{gathered}
|P(x, t)| \leq\left(\frac{t}{x}\right)^{\beta+1} \frac{2^{\beta-2}}{\beta}\left[\frac{t^{2}+x^{2}}{2 t x}\right]^{\beta} \exp \left[\left(\frac{t-x}{2}\right) \frac{2^{\beta-2}}{\beta}\left[\frac{t^{2}+x^{2}}{2 t r}\right]^{\beta}\right] \\
=\frac{1}{4 \beta} \frac{1}{x^{2 \beta+1}}\left(t^{2}+x^{2}\right)^{\beta} \exp \left[\frac{2^{\beta-2}}{\beta}\left(\frac{t-x}{2}\right)\left(\frac{t^{2}+x^{2}}{2 t x}\right)^{\beta}\right] .
\end{gathered}
$$

Proof. In this case, we transform the estimate from Theorem 3 to

$$
\begin{gathered}
\frac{\Gamma(\beta) 4^{\beta-1}}{\left(t^{2}-x^{2}\right)^{\beta}} P_{\beta}^{-\beta}\left(\frac{t^{2}+x^{2}}{2 t x}\right)=\frac{\Gamma(\beta) 4^{\beta-1}}{\left(t^{2}-x^{2}\right)^{\beta}} \frac{2^{-\beta}}{\Gamma(\beta+1)}\left[\left(\frac{t^{2}+x^{2}}{2 t x}\right)^{2}-1\right]^{\beta / 2} \\
=\frac{2^{\beta-2}}{\beta} \frac{1}{\left(t^{2}-x^{2}\right)^{\beta}} \frac{\left(t^{2}-x^{2}\right)^{\beta}\left(t^{2}+x^{2}\right)^{\beta}}{(2 t x)^{\beta}}=\frac{2^{\beta-2}}{\beta}\left[\frac{t^{2}+x^{2}}{2 t x}\right]^{\beta},
\end{gathered}
$$

using in the transformation the formula (see [20])

$$
P_{v}^{-v}(z)=\frac{2^{-v}}{\Gamma(v+1)}\left(z^{2}-1\right)^{v / 2}, \quad z>1
$$

Therefore, the inequality for the kernel for $\beta=\alpha-1$ takes the form (20).

The corollary is proved.

Note that, for $\alpha=0$ in formulas (1) and (2), Theorem 1 reduces to the well-known estimates for the kernel of the Levin-type integral representation for the Sturm-Liouville equation.

The technique presented above can be fully transferred to the problem of constructing non-classical generalized shift operators. This problem is essentially equivalent to expressing solutions to the equation

$$
B_{\alpha, x} u(x, y)-q(x) u(x, y)=B_{\beta, y} u(x, y)
$$

via the solutions of the unperturbed Euler-Poisson-Darboux equation with Bessel operators in each variable (in the non-singular case, the wave equation) with additional conditions ensuring well-posedness. Such representations are obtained from the mere fact of the existence of transform operators and were studied for the non-singular case $(\alpha=\beta=0)$ in [22, 23] as an implication of the theory of generalized shift. An interesting original technique for obtaining such representations, also in a non-singular case, was developed by Borovskikh [24]. The results of the present work imply integral representations of a certain subclass of solutions of Eq. (22) in the general singular case for quite arbitrary potentials with singularities at the origin. Moreover, the estimates for the solutions do not contain any indefinite constants and, for the kernels of integral representations, integral equations satisfied by them are written out explicitly.

\section{ASYMPTOTICALLY EXACT INEQUALITIES FOR THE LEGENDRE FUNCTIONS}

In conclusion, let us show how one can use formula (18) obtained when deriving Lemma 5 to establish an asymptotically exact lower bound for the Legendre functions. In the author's opinion, this estimate is of independent interest.

Consider the obvious inequalities $(\alpha>0)$

$$
\int_{0}^{1} P_{v}(1+2 \alpha x)(1-x)^{-1 / 2} d x \leq \int_{0}^{1} P_{v}(1+2 \alpha x) x^{-1 / 2}(1-x)^{-1 / 2} d x \leq P_{v}(1+2 \alpha) \int_{0}^{1} x^{-1 / 2}(1-x)^{-1 / 2} d x .
$$


The first integral is obtained from formula (18) for $\beta=\frac{1}{2}$; it was calculated in Lemma 5 . The second integral is known, and, as follows, e.g., from [35], is calculated by the formula

$$
I=\pi^{1 / 2} \Gamma\left(\frac{1}{2}\right)\left\{P_{v}(\sqrt{1+\alpha})\right\}^{2} .
$$

Therefore, we have the inequality

$$
\sqrt{\pi} \alpha^{-1 / 4}(\alpha+1)^{-1 / 4} P_{v}^{-1 / 2}(2 \alpha+1) \leq \pi\left\{P_{v}(\sqrt{1+\alpha})\right\}^{2} .
$$

Expressing the Legendre function in terms of elementary functions, we finally obtain

$$
\left\{P_{v}(\sqrt{1+\alpha})\right\}^{2} \geq \frac{1}{\pi(2 v+1) \sqrt{x}}\left\{(\sqrt{x}+\sqrt{x+1})^{2 v+1}-(\sqrt{x}+\sqrt{x+1})^{-2 v-1}\right\} .
$$

The inequality just proved gives correct asymptotics for all parameters entering into it, as follows from the asymptotic formula presented in [26]:

$$
\left[P_{v}(\sqrt{1+x})\right]^{2} \sim \frac{1}{2 \pi v} \frac{(\sqrt{x}+\sqrt{x+1})^{2 v+1}}{\sqrt{x}} .
$$

The second of the integral inequalities considered also leads to an interesting estimate

$$
\left\{P_{v}(\sqrt{1+\alpha})\right\}^{2} \leq P_{v}(1+2 \alpha) .
$$

In connection with the last inequalities presented, it should be noted that the Legendre functions are related to the complete and incomplete elliptic Legendre integrals of three kinds; these two classes of special functions are expressed, at certain values, via each other. On the other hand, the Legendre functions are special cases of the Gauss hypergeometric function. Various inequalities for the Legendre elliptic integrals are considered in the author's joint works [27, 28], and inequalities for hypergeometric functions, in $[29,30]$.

\section{ACKNOWLEDGMENTS}

I am grateful to A.V. Glushak (Belgorod State University) and V.V. Kravchenko (CINVESTAV, Queretaro, Mexico) for useful comments, which made it possible to clarify the formulation of the problem under consideration.

\section{REFERENCES}

1. R. Carroll, Transmutation, Scattering Theory, and Special Functions (Elsevier, Amsterdam, 1982).

2. R. Carroll, Transmutation Theory and Application (Elsevier, Amsterdam, 1986).

3. V. A. Marchenko, Sturm-Liouville Operators and Applications (Kiev, Naukova Dumka, 1977; Birkhäuser, Basel, 1986).

4. B. M. Levitan, Inverse Sturm-Liouville Problems (Nauka, Moscow, 1984; VNU Science, Utrecht, 1987).

5. V. V. Katrakhov and S. M. Sitnik, "The transmutation method and boundary value problems for singular elliptic equations," Sovrem. Mat. Fundam. Napravlen. 64 (2), 211-426 (2018).

6. S. M. Sitnik and E. L. Shishkina, Transmutation Method for Differential Equations with Bessel Operators (Fizmatlit, Moscow, 2019) [in Russian].

7. K. Chadan and P. C. Sabatier, Inverse Problems in Quantum Scattering Theory (Springer-Verlag, New York, 1977).

8. R. O. Hryniv and Ya. V. Mykytyuk, "Transformation operators for Sturm-Liouville operators with singular potentials: Dedicated to Professor V.A. Marchenko on the occasion of his 80th birthday," Math. Phys. Anal. Geom. 7, 119-149 (2004).

9. B. M. Levitan, "Expansion in Fourier series and integrals with Bessel functions," Usp. Mat. Nauk 6 (2), 102143 (1951).

10. V. V. Stashevskaya, "Inverse problem of spectral analysis for a differential operator with singularity at zero," Uch. Zap. Kharkov. Mat. Ob-va, No. 5, 49-86 (1957).

11. A. S. Sokhin, "On a class of transformation operators," Tr. Fiz.-Tekh. Inst. Nizkikh Temp. Akad. Nauk Ukr. SSR, No. 1, 117-125 (1969).

12. A. S. Sokhin, "Transformation of operators for equations with a special singularity," Vestn. Kharkov. Univ. 113, 36-42 (1974). 
13. V. V. Kravchenko, “A representation for solutions of the Sturm-Liouville equation,” Complex Variables Elliptic Equations 53, 775-789 (2008).

14. V. V. Kravchenko and R. M. Porter, "Spectral parameter power series for Sturm-Liouville problems," Math. Method Appl. Sci. 33, 459-468 (2010).

15. V. V. Kravchenko, "On a method for solving the inverse scattering problem on the line," Math. Methods Appl. Sci. 42, 1321-1327 (2019).

16. V. V. Kravchenko, "On a method for solving the inverse Sturm-Liouville problem," J. Inverse Ill-Posed Probl. 27, 401-407 (2019).

17. S. M. Sitnik, Preprint IAPU DVO RAN (Institute of Automation and Control Processes, Far Eastern Branch, Russian Academy of Sciences, Vladivostok, 1993).

18. V. V. Katrakhov and S. M. Sitnik, "Estimates of the Jost solution to a one-dimensional Schrodinger equation with a singular potential," Dokl. Math. 51 (1), 14-16 (1995).

19. A. A. Kilbas, O. I. Marichev, and O. A. Repin, Boundary Value Problems for Partial Differential Equations with Discontinuous Coefficients (Samar. Gos. Ekon. Univ., Samara, 2008) [in Russian].

20. Higher Transcendental Functions (Bateman Manuscript Project), Ed. by A. Erdélyi (McGraw-Hill, New York, 1953), Vol. 1.

21. O. I. Marichev, Handbook of Integral Transforms of Nigher Transcendental Functions: Theory and Algorithmic Tables (Horwood, Chichester, England, 1983; Nauka i Tekhnika, Minsk, 1978).

22. B. M. Levitan, "The application of generalized displacement operators to linear differential equations of the second order," Usp. Mat. Nauk 4 (1), 3-112 (1949).

23. B. M. Levitan, Generalized Translation Operators and Some of Their Applications (Fizmatgiz, Moscow, 1962; Israel Program for Scientific Translations, Jerusalem, 1964).

24. A. V. Borovskikh, "Method of propagating waves for a one-dimensional inhomogeneous medium," J. Math. Sci. 127, 2135-2158 (2005).

25. Tables of Integral Transforms (Bateman Manuscript Project), Ed. by A. Erdélyi (McGraw-Hill, New York, 1954), Vol. 1.

26. G. Pólya and G. Szegö, Aufgaben und Lehrsätze aus der Analysis (Springer-Verlag, Berlin, 1964).

27. D. B. Karp and S. M. Sitnik, "Asymptotic approximations for the first incomplete elliptic integral near logarithmic singularity,” J. Comput. Appl. Math. 205, 186-206 (2007).

28. D. Karp, A. Savenkova, and S. M. Sitnik, "Series expansions for the third incomplete elliptic integral via partial fraction decompositions," J. Comput. Appl. Math. 207 (2), 331-337 (2007).

29. D. Karp and S. M. Sitnik, "Inequalities and monotonicity of ratios for generalized hypergeometric function," J. Approximation Theory 161, 337-352 (2009).

30. D. B. Karp and S. M. Sitnik, "Log-convexity and log-concavity of hypergeometric-like functions," J. Math. Appl. 364, 384-394 (2010).

Translated by E. Chernokozhin 